\title{
Subdivision Curves on Surfaces and Applications
}

\author{
Dimas Martínez Morera, Luiz Velho, and Paulo Cezar Carvalho \\ Instituto Nacional de Matemática Pura e Aplicada-IMPA, Brazil
}

\begin{abstract}
Subdivision curves have great importance for many CAD/ CAM applications.. In this paper we propose a simple method to define subdivision schemes on triangulations. It works by translating to the triangulation a perturbation of a planar binary subdivision. To reproduce this perturbation in the surface we use both, shortest and straightest geodesics, so we call this strategy intrinsic projection method. It can reproduce any binary subdivision scheme, regardless whether it is linear or not.
\end{abstract}

Keywords: Subdivision Curve, Discrete Geodesic, Manifold Triangulation.

\section{Introduction}

Subdivision curves are very important for several science and engineering applications. In the Euclidean space $\mathbb{R}^{n}$, they are easy to define by just selecting a small set of control points. A drawing algorithm typically performs some subdivision steps, thus approximating the limit curve by a polygonal line in a matter of milliseconds. In this setting, there are many references on the convergence of these schemes.

Given their good properties and the advantages of using these curves, it is natural to extend them to non-euclidean geometries, as Riemannian manifolds, Lie groups or triangulations. In section 3 we review some of these extensions. In this paper we propose a simple method to define subdivision schemes on triangulations.

\subsection{Overview}

The paper is organized as follows: In section 2 we make a short review of subdivision curves on the euclidean space. Section 3 begins reviewing the different extensions of subdivision schemes to non-euclidean geometries, and the main contributions of this work are lately presented in this section, where we propose a general method to define subdivision schemes on triangulations. We comment an application of subdivison curves to mesh segmentation in section 4, while in section 5 we conclude with some remarks about this work.

\section{Subdivision Curves}

Subdivision schemes constitute an efficient tool for curve and surface design in Computer Graphics. In this section we make a brief review of subdivision 
schemes for curve design on $\mathbb{R}^{d}$. Given a polygonal line $P$, with vertices on $\mathbb{R}^{d}$ denoted by $\cdots, P_{-1}, P_{0}, P_{1}, \cdots$, a subdivision of $P$ is defined as the limit of a sequence of refinements of it. For the sake of simplicity, we asume that polygons have infinitely many vertices. In practice, however, care must be taken when dealing with open polygonal lines due to the lack of neighbors for vertices close to first and last vertices of the polygonal.

The binary subdivision of $P^{0}$ (namely $P^{1}$ ) is obtained by first inserting a new point between two consecutive points of $P^{0}$ and then perturbing the position of some or all the points. The rules used to perturb the points are determined by the subdivision scheme. If we denote by $S$ the refinement operator then we have $P^{j+1}=S P^{j}=S^{j} P^{0}=S^{j} P$ and the limit curve is denoted by $S^{\infty} P$.

Subdivision schemes are classified according to the rules used to obtain $P^{j+1}$ from $P^{j}$. Note that new vertices in $P^{j+1}$ have odd indexes while old vertices correspond to even indexes in $P^{j+1}$. It is then natural to have different rules for even and odd vertices:

$$
\begin{aligned}
& P_{2 i}^{j+1}=\cdots+a_{-2} P_{i+1}^{j}+a_{0} P_{i}^{j}+a_{2} P_{i-1}^{j}+\cdots \\
& P_{2 i+1}^{j+1}=\cdots+a_{-1} P_{i+1}^{j}+a_{1} P_{i}^{j}+a_{3} P_{i-1}^{j}+\cdots
\end{aligned}
$$

In these two equations, the sequence of coefficients $a=\left\{a_{i}, i \in \mathbb{Z}\right\}$ is called mask and is supposed to have only a finite number of non-zero entries. These rules can be summarized in one equation,

$$
P_{i}^{j+1}=\left(S P^{j}\right)_{i}=\sum_{k \in \mathbb{Z}} a_{i-2 k} P_{k}^{j}, \quad i \in \mathbb{Z}
$$

A deeper study of linear stationary schemes can be found in the book of Cavaretta et. al. 2]. A collection of non-linear schemes was described by Dyn and Levin 44.

\subsection{Examples}

Chaikin's Scheme. Maybe the most intuitive way to smooth a polygonal line is to cut its corners. Chaikin's scheme does exactly that. Each refinement consists on cutting the consecutive edges of the polygon in the ratio $\frac{1}{4}: \frac{1}{2}: \frac{1}{4}$. Figure 1(a) shows some steps of the refinement of a polygon using Chaikin's rule. The following are the equations of this scheme:

$$
P_{2 i}^{j+1}=\frac{3}{4} P_{i}^{j}+\frac{1}{4} P_{i+1}^{j}, \quad P_{2 i+1}^{j+1}=\frac{1}{4} P_{i}^{j}+\frac{3}{4} P_{i+1}^{j} . .
$$

Schemes like Chaikin's are called approximant because they do not interpolate the vertices of the control polygon, so that the resulting curves are just smooth approximations of the control polygon.

4-point Scheme. Interpolant schemes are those which interpolate the vertices of the control polygon. Consequently, every new vertex belongs to the curve 


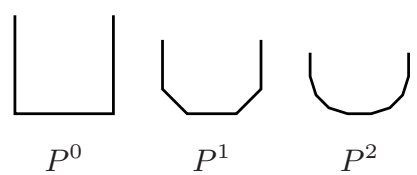

(a)

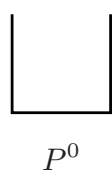

$P^{0}$

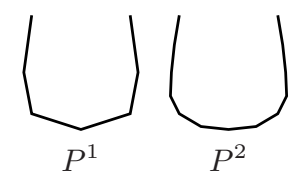

(b)

Fig. 1. Refinement of a polygon using (a) Chaikin's scheme, and (b) 4-point scheme

$S^{\infty} P$. The more popular interpolant scheme is the so-called 4-point scheme [5]. Its rules are:

$$
P_{2 i}^{j+1}=P_{i}^{j}, \quad P_{2 i+1}^{j+1}=\left(\frac{1}{2}+w\right)\left(P_{i}^{j}+P_{i+1}^{j}\right)-w\left(P_{i-1}^{j}+P_{i+2}^{j}\right)
$$

where $w$ is a tension parameter. Figure1(b) shows some steps of the 4-point scheme.

\section{Subdivision Curves on Manifolds}

The problem of designing curves on manifolds is very interesting and have various applications. Some variants of de Casteljau's Algorithm 3] have been applied to define curves on Riemannian manifolds and Lie groups [1013], and also on triangulations [7. Hofer and Pottmann [12] defined spline curves by minimizing some energy functionals. Bonneau and Hahmann [1] give some strategies to smooth curves on triangulations.

Recently, a general framework for linear subdivision on smooth manifolds have been described by Helmut and Pottmann [18]. The convergence and smoothness analysis of this curves can be found in the works of Wallner and Dyn 16[17. They generalize the linear schemes to manifolds in two different ways. Their first method is to substitute linear average by geodesic average. This method is very good because it is completely intrinsic although for some schemes it would have to compute many geodesics. The second method is to perform each subdivision step in the ambient space, projecting the new points into the manifold.. This approach is more efficient but depending on the geometry could conduce to wrong or unexpected results.

We propose a third method to generalize subdivision schemes to manifolds. It uses geodesic averages but it is not based on them, so it can reproduce non-linear schemes as well, it also performs fewer geodesic computations. In the other hand, it does a sort of intrinsic projection that eliminates the problem of getting the wrong projection when the surface has a complicated geometry.

\subsection{Revisiting Curve Subdivision}

Consider the following subdivision scheme:

$$
P_{2 i}^{j+1}=P_{i}^{j}, \quad P_{2 i+1}^{j+1}=\frac{\left(P_{i}^{j}+P_{i+1}^{j}\right)}{2} .
$$


This scheme performs a linear subdivision consisting just on inserting new points at midpoints of each pair of consecutive old points. Lets call this scheme of $B$.

Every binary subdivision scheme can be written as a perturbation of $B$, regardless of it being linear or not. We have now a uniform description for all binary schemes. For example, given a scheme $S$ and a set of control points $P$, defining $v_{i}=(S P)_{i}-(B P)_{i}$ we can write

$$
(S P)_{i}=(B P)_{i}+v_{i}
$$

Now each subdivision of a control polygon can be regarded as a two step process, where we perform one step of subdivision given by $B$, and one step of perturbation given by the set of vectors $v_{i}$.

This approach to subdivision schemes may not be of great use in the case of traditional schemes on the euclidean space, particularly linear schemes. However, it will be very useful to reproduce planar subdivision schemes on manifolds.

\subsection{Intrinsic Projection Method}

Since triangle meshes are manifolds of dimension two, we are going to define our subdivision schemes by imitating planar schemes. Given a triangle mesh $\mathcal{M}$, a control polygon $P$ with vertices on $\mathcal{M}$, and a subdivision scheme $S$, we define the scheme $S_{\mathcal{M}}$ as a two step process as done above.

Step 1: binary subdivision. To perform this step we use the scheme $B_{\mathcal{M}}$, defined similar to $B$ :

$$
P_{2 i}^{j+1}=P_{i}^{j}, \quad P_{2 i+1}^{j+1}=\operatorname{midpoint} \text { of } \operatorname{geodesic}\left(P_{i}^{j}, P_{i+1}^{j}\right) .
$$

The difference between this scheme and $B$ is the substitution of linear interpolation by geodesic interpolation. See section 3.3 for a review of discrete geodesic computation.

Step 2: perturbation. To perform the perturbation step we need to compute the vectors $v_{i}$. Also the resulting point $\left(S_{\mathcal{M}} P\right)_{i}=\left(B_{\mathcal{M}} P\right)_{i}+v_{i}$ must belong to $\mathcal{M}$. It is very hard to fulfill such restriction, so we proceed in a different way. To compute the perturbation we find first a vector $t_{i}$ tangent to $\mathcal{M}$ at $\left(B_{\mathcal{M}} P\right)_{i}$. After that, the new point $\left(S_{\mathcal{M}} P\right)_{i}$ belongs to the unique straightest geodesic line departing from $\left(B_{\mathcal{M}} P\right)_{i}$ in the direction of $t_{i}$ and is distant to $\left(B_{\mathcal{M}} P\right)_{i}$ by the magnitude of $t_{i}$. See section 3.3 for references on discrete tangent vectors and the unicity of straightest geodesic.

Rest now to compute the vectors $t_{i}$. The idea is simple, we are going to create a plane control polygon $\tilde{P}$ whose sides have the same length as the sides (geodesic curves) of $P$, and whose angles are proportional to those of $P$. If a vertex of $P$ coincides with a mesh vertex, the total angle $\alpha$ at that mesh vertex will 
probably be different from $2 \pi$, so the corresponding angle in $\tilde{P}$ will be proportional to it. For instance, if the angle of $P$ at that vertex is $\beta$, the corresponding angle at $\tilde{P}$ will be $(2 \pi / \alpha) \beta$. For vertices of $P$ inside a face or edge of $\mathcal{M}$ we have $\alpha=2 \pi$ so the angle in $\tilde{P}$ will be the same. To compute the vectors $t_{i}$ we first compute the $v_{i}$ 's using (6) for $\tilde{P}$ :

$$
v_{i}=(S \tilde{P})_{i}-(B \tilde{P})_{i} .
$$

Now $t_{i}$ is obtained from $v_{i}$ by changing the coordinates to the tangent plane to $\mathcal{M}$ at $P_{i}$.

\subsection{Implementation}

The implementation of the intrinsic projection method is very simple. In this section we do a small description of the details involved, and show the results of the implementation of Chaikin and 4-point schemes.

To perform the binary subdivision step on the polygon $P$, we just compute the shortest geodesic line joining every two consecutive vertices of $P$, and insert its midpoint between the two points. Thus obtaining $B_{\mathcal{M}} P$.

To perform the perturbation step, for each vertex $\left(B_{\mathcal{M}} P\right)_{i}$, take the portion of $P$ that influence it and construct a plane polygon $\tilde{P}_{i}$, and compute the corresponding plane perturbation vector $v_{i}$. Observe that the amount of vertices of $P$ influencing a vertex of $B_{\mathcal{M}} P$ depends on the subdivision scheme.

The more delicate part is the mapping of $v_{i}$ to a tangent vector $t_{i}$. We proceed as follows:

$\boldsymbol{v}_{\boldsymbol{i}}$ : Let $v$ be the vector $\tilde{P}_{i+1}-\tilde{P}_{i}$. Vector $v_{i}$ is given as a pair $\left(\rho_{i}, l_{i}\right)$, where $\rho_{i}$ is the anticlockwise angle between the vectors $v$ and $v_{i}$, and $l$ is the size of $v_{i}$.

$\boldsymbol{t}_{\boldsymbol{i}}$ : To obtain $t_{i}$, find the direction at $\left(B_{\mathcal{M}} P\right)_{i}$ on $M$ that makes an anticlockwise angle proportional to $\rho_{i}$ with the geodesic segment joining $\left(B_{\mathcal{M}} P\right)_{i}$ and $\left(B_{\mathcal{M}} P\right)_{i+1}$.

perturbation: The perturbation is given by the extreme of the straightest geodesic beginning at $\left(B_{\mathcal{M}} P\right)_{i}$ in the direction of $t_{i}$, whose length is $l_{i}$.

Discrete Geodesics. We are interested on designing curves on triangulations, so we need to compute discrete geodesics. Locally shortest curves [8,9]5] solve the boundary value problem, whereas straightest geodesics [11 solve the initial value problem. In this setting, a direction is given as a tangent vector to the surface; the definition of tangent vector for triangulated surfaces [1] can be summarized in the following

Definition 1. A vector $v$ is tangent to the mesh $\mathcal{M}$ at point $P$, if there exists a positive number $\varepsilon$ such that the point $P+\varepsilon v$ belongs to a face $f$ containing $P$.

Note that -by the previous definition- the set of all tangent vectors to $\mathcal{M}$ at $P$ do not necessarily belongs to a plane. This happens exactly when $P$ lies in an edge or vertex of $\mathcal{M}$. In those cases, the tangent vectors belong to the support planes of the faces incident to $P$. 
Chaikin's Scheme. The Chaikin's scheme has the particularity that every time we compute a $v_{i}$ vector, its associated angle $\rho_{i}$ equals 0 . Also $l_{i}=0.25 l$, where $l$ is the length of the corresponding shortest geodesic segment.. In this particular case, we can substitute the old points by the points corresponding to geodesic interpolation with parameters 0.25 and 0.75 . Figure 2 shows the first subdivision steps of a polygon, using Chaikin's scheme.
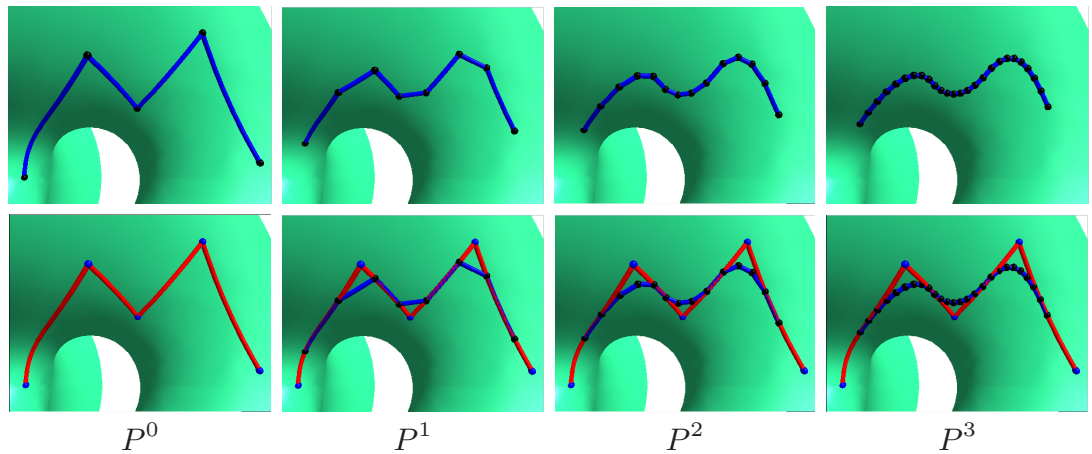

Fig. 2. First steps of Chaikin's subdivision scheme. The control polygon are also shown in the second line. Black dots indicate the vertices of the refined polygon.

4-point Scheme. Since the 4-point scheme is interpolant, old points does not need to be perturbed. For the perturbation of each new point we must use four old points. Using these old points we can compute the planar perturbation $(\rho, l)$ of $B$, and translate it to the $\mathcal{M}$ using straightest geodesics. Figure 3 shows the first subdivision steps of a polygon, using the 4-point scheme.
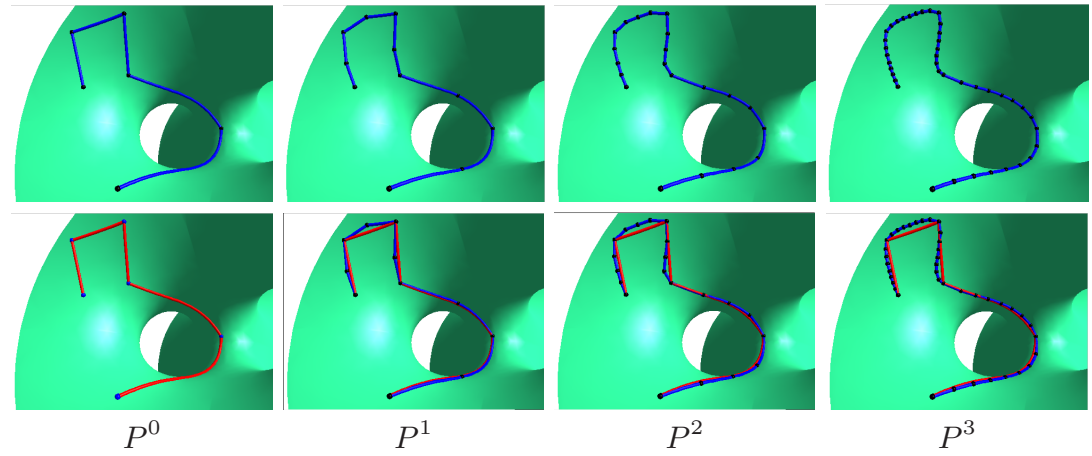

Fig. 3. First steps of 4-point subdivision scheme. The control polygon are also shown in the second line. Black dots indicate the vertices of the refined polygon. 


\section{Mesh Segmentation with Subdivision Curves}

In some problems related to $3 \mathrm{D}$ shape analysis, it is very important to segment a surface 6 614. Feature lines are the natural candidates to boundary of the segmented regions. Subdivision curves provide a flexible tool to describe those feature lines, since few points are sufficient to describe the lines. Besides, a potencial user could modify them locally, perfoming a fine tunning of the shape of the final curve. The smooth appearance of the lines thus obtained, improves the visual quality of the segmentation and contributes for further processing of the segmented meshes. See Fig. 4 for a simple example of the segmentation of a cube, where the feature lines were extracted based on the discrete gaussian curvature.

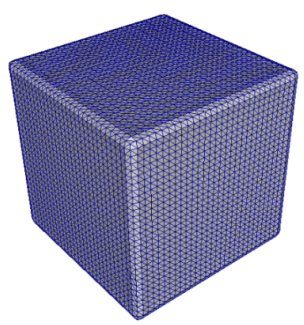

(a)

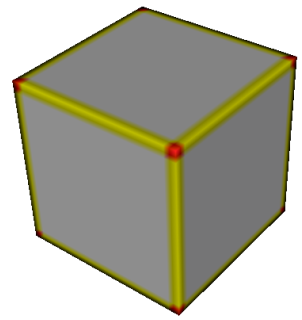

(b)

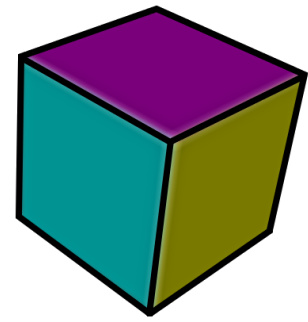

(c)

Fig. 4. Segmenting a cube: (a) the original mesh, (b) discrete gaussian curvature plot and (c) cube segmented using subdivision curves to describe the feature lines

\section{Conclusion}

We have presented the intrinsic projection method to reproduce planar subdivision schemes on manifold triangulations. Since this method is based on planar schemes, it coincides with those schemes on planar meshes. Its extensions to smooth manifolds is straightforward. A nice feature of the method is its capability of reproducing non-linear schemes in the same way as the linear case.

The intrinsic projection method can be easily adapted to smooth manifolds. It is possible to compute geodesics solving both, the boundary value and initial value problem on those manifolds. See [18] for instance. In this context would be very nice to study the smoothness properties of $S^{\infty} P$.

\section{Acknowledgments}

This research has been developed in the VISGRAF Laboratory at IMPA. VISGRAF Laboratory is sponsored by CNPq, FAPERJ, FINEP and IBM Brazil. 


\section{References}

1. Bonneau, G., Hahmann, S.: Smooth polylines on polygon meshes. In: Mathematics and Visualization, pp. 69-84. Springer, Heidelberg (2004)

2. Cavaretta, A.S., Dahmen, W., Micchelli, C.A.: Stationary Subdivision. American Mathematical Society, Boston (1991)

3. de Casteljau, P.F.: Outillage Méthodes Calcul. Internes Dokument P2108, SA André Citroën, Paris (February 1959)

4. Dyn, N., Levin, D.: The subdivision experience. In: Laurent, P.J., Le M'ehaut'e, A., Schumaker, L.L. (eds.) Wavelets, Images and Surface Fitting, pp. 229-244. A K Peters, Ltd., Wellesley (1994)

5. Dyn, N., Levin, D., Gregory, J.A.: A 4-point interpolatory subdivision scheme for curve design. Computer Aided Geometric Design 4, 257-268 (1987)

6. Marinov, M., Kobbelt, L.: A robust two-step procedure for quad-dominant remeshing. Computer Graphics Forum 25(3), 537-546 (2006)

7. Martínez, D., Carvalho, P.C., Velho, L.: Geodesic Bézier curves: A tool for modeling on triangulations. In: Proceedings of SIBGRAPI 2007. XX Brazilian Symposium on Computer Graphics and Image Processing. XX Brazilian Symposium on Computer Graphics and Image Processing, pp. 71-78. IEEE Computer Society, Los Alamitos (2007)

8. Martínez, D., Velho, L., Carvalho, P.C.: Computing geodesics on triangular meshes. Computer and Graphics 29(5), 667-675 (2005)

9. Mitchell, J.S.B., Mount, D.M., Papadimitriou, C.H.: The discrete geodesic problem. SIAM J. Comput. 16, 647-668 (1987)

10. Park, F.C., Ravani, B.: Bezier curves on Riemannian manifolds and Lie groups with kinematic applications. ASME Journal of Mechanical Design 117, 36-40 (1995)

11. Polthier, K., Schmies, M.: Straightest geodesics on polyhedral surfaces. In: Hege, H.-C., Polthier, K. (eds.) Visualization and Mathematics, pp. 135-150. Springer, Heidelberg (1998)

12. Pottmann, H., Hofer, M.: A variational aproach to spline curves on surfaces. Computer Aided Geometric Design 22(7), 693-709 (2005)

13. Rodriguez, R.C., Leite, F.S., Jacubiak, J.: A new geometric algorithm to generate smooth interpolating curves on riemannian manifolds. LMS Journal of Computation and Mathematics 8, 251-266 (2005)

14. Rössl, C., Kobbelt, L., Seidel, H.: Extraction of feature lines on triangulated surfaces using morphological operators. In: Proceedings of Smart Graphics, Stanford, USA, pp. 71-75. AAAI Press, Menlo Park (2000)

15. Surazhsky, V., Surazhsky, T., Kirsanov, D., Gortler, S., Hoppe, H.: Fast exact and approximate geodesics on meshes. In: Proceedings of ACM SIGGRAPH 2005, pp. 553-560 (2005)

16. Wallner, J.: Smoothness analysis of subdivision schemes by proximity. Constr. Approx. 24(3), 289-318 (2006)

17. Wallner, J., Dyn, N.: Convergence and $C^{1}$ analysis of subdivision schemes on manifolds by proximity. Comput. Aided Geom. Design 22(7), 593-622 (2005)

18. Wallner, J., Pottmann, H.: Intrinsic subdivision with smooth limits for graphics and animation. ACM Trans. Graphics 25(2), 356-374 (2006) 